\title{
Physiological Responses under Drought Stress of Improved Drought- Tolerant Rice Lines and their Parents
}

\author{
Preeyanuch LARKUNTHOD ${ }^{1}$, Noppawan NOUNJAN ${ }^{1}$, Jonaliza L. \\ SIANGLIW ${ }^{2,3}$, Theerayut TOOJINDA ${ }^{2,3}$, Jirawat SANITCHON ${ }^{4}$, Boonrat \\ JONGDEE ${ }^{4}$, Piyada THEERAKULPISUT ${ }^{1 *}$
}

\author{
${ }^{1}$ Khon Kaen University, Faculty of Science, Department of Biology, Salt-tolerant Rice Research Group, Khon Kaen 40002, \\ Thailand;preeyanut@kkumail.com;nounjann@gmail.com;piythe@kku.ac.th (*correspondingauthor) \\ ${ }^{2}$ Rice Gene Discovery Unit, BIOTEC, NSTDA, Kasetsart University, Kamphangsaen, Nakhon Pathom 73140, Thailand; jsiangliw@gmail.com \\ ${ }^{3}$ Plant Biotechnology Research Unit, BIOTEC, NSTDA 113 Thailand Science Park, Khlong Nueng, Khlong Luang, Pathum Thani 12120, \\ Thailand; toojindatheerayut@gmail.com \\ ${ }^{4}$ Khon Kaen University, Faculty of Agriculture, Department of Plant Science and Agricultural Resources, \\ Khon Kaen 40002, Thailand; jirawat@kku.ac.th; boonrat.j@rice.mail.go.th
}

\begin{abstract}
Many of the economically important rice cultivars including 'Khao Dawk Mali 105' (KDML105) or jasmine rice, one of the world's famous rice exported from Thailand suffers from drought due to erratic rainfalls and limited irrigation. To improve drought tolerance and reserve genetic background of KDML105, chromosome segment substitution lines (CSSL) containing drought tolerant quantitative trait loci (DT-QTL) has been previously developed by backcrossing between KDML105 and drought tolerant donor, IR58586-F2-CA-143 (DH212). To understand the physiological responses related to drought tolerance in CSSL lines compared to parents, two CSSLs namely CSSL1-16 and CSSL1-18, respectively were used in this study. Twenty-one-d-old hydroponically grown plants were subjected to $20 \%$ PEG for $0,7,14 \mathrm{~d}$ and then recovered from stress for $3 \mathrm{~d}$. The results indicated that CSSL lines especially, CSSL1-16 showed better performance under drought stress compared to their recurrent parent. Drought tolerance superior CSSL1-16 line was indicated by high water status (high relative water content and leaf water potential), good osmotic adjustment, high proline and greater membrane stability. Moreover, this line was able to resume growth after stress recovery whereas other lines/cultivar could not recover. Similarly, drought tolerant donor showed high water status suggesting that well-maintained plant water status was associated with drought tolerant trait. It could be concluded that the highest drought tolerant line was CSSL1-16 followed by DH212, CSSL1-18 and KDML105. It would be interesting to go further into introgressed section in CSSL1-16 to identify potential candidate genes in DT-QTL for breeding drought tolerant rice in the future.
\end{abstract}

Keywords: drought stress; drought tolerance QTL; leaf water potential; osmotic adjustment; rice

\section{Introduction}

In recent years, agricultural areas worldwide are affected by drought. Drought imposes serious influences on growth and development of plants by causing numerous changes at the physiological, metabolic and molecular levels ( $\mathrm{Zu}$ et al., 2017). Plants respond variously to drought stress in terms of morphology and physiology under drought conditions. Different mechanisms allow plants to survive and even reproduce with a limited water supply, such as the maximization of water uptake by deep, dense root systems, the minimization of water loss by stomatal closure and a reduction in leaf area, osmotic adjustment $(\mathrm{OA})$ or changes in cell wall elasticity as well as other essential processes for maintaining physiological activities throughout extended periods of drought (Saha et al., 2016). OA is regarded as an important mechanism to keep osmotic potential (OP) inside the plant cells to be lower than outside the cells, thus preventing water loss and allowing plants to resume their turgid pressure. Accumulation of organic substances and inorganic ions such as sucrose, glucose, proline and potassium ions by plants exposed to water stress is believed to be a mechanism participating in $\mathrm{OA}$ which could 
680

promote drought stress tolerance in plants (Zivcak et al., 2016). Plants exposed to almost all kinds of abiotic stresses including drought leads to elevated oxidative stress with overproduction of reactive oxygen species (ROS), which are highly toxic and cause damage to proteins, lipids, carbohydrates, and DNA (Farooq et al., 2011). It has been well documented that drought tolerant plants had higher efficiency in protection of plant cells from ROS via producing antioxidant enzymes and non-enzymatic antioxidants (Farooq et al., 2012).

As plant response to drought stress varies across genotypes and is strongly affected by environment and genotype by environment interaction, the use of physiological traits as an indirect selection would be important in augmenting yield-based selection procedures (Lonbani and Arzani, 2011). Current rice production systems rely on an ample water supply and thus are more vulnerable to drought stress. Drought is the most important limiting factor for rice production and is becoming an increasingly severe problem (Bouman et al., 2005). Most of the rice varieties preferred by farmers in tropical and subtropical areas are susceptible to drought stress ( $\mathrm{Zu}$ et al., 2017). Rice is an important economic crop in Thailand, which is mainly cultivated in rainfed lowland areas of the country, where the cultivation relies largely on seasonal rainfall. The majority of rice-growing areas, especially the northeastern regions, often encounter problems associated with unpredictable quantity and distribution of precipitation. As most rice-producing areas are sandy soils that lack water retention, the occurrence of dry spell results in dramatically lowered water level and soil humidity insufficient to meet plant water demands (Jongdee et al., 2002).

Thai aromatic rice namely 'Khao Dawk Mali 105' (KDML105) or jasmine rice is a drought susceptible cultivar. Developing improved lines of KDML105 rice with tolerance to drought is an interesting approach for sustainable solution to solve low KDML105 productivity in the northeastern part of the country. To improve drought tolerance and reserve genetic background determining good traits of KDML105 (such as soft texture, jasmine-like aroma and good cooking/eating quality), chromosome segment substitution lines (CSSL) containing drought tolerant quantitative trait loci (DT-QTL) in various segments had been developed by marker-assisted backcrossing between KDML105 and drought tolerant donor, IR58586-F2-CA-143 (DH212) (Kanjoo et al., 2012). This CSSL population (KDML105 CSSL) was evaluated for drought tolerance. It was found that these CSSLs had better grain yield and yield components than KDML105. In addition, it was revealed that QTL associated with grain yield and yield components were located on chromosomes 1, 3, 4, 8 and 9 (Kanjoo et al., 2012). To clarify the characteristics of KDML105CSSL population under drought stress, the physiological responses of two selected KDML105CSSL lines which received DTQTL segments on chromosome 1 namely RGD05164MAS67-MAS10-B-B-B-B with code CSSL1-16 and RGD05164-MAS75-MAS18-B-B-B-B with code CSSL118 , were investigated and compared to the parents.

\section{Materials and Methods}

\section{Plant materials, growth conditions and drought treatment}

Two CSSLs and parents (KDML105 and IR58586-F2CA-143 (DH212)) were used in this experiment. Seeds were surfaced sterilized by soaking in 5\% sodium hypochlorite for $30 \mathrm{~min}$ and washed several times with distilled water. Seeds were germinated on filter paper and germinated seedlings were transferred to a hydroponic system where plants were grown on plastic grids floated on aerated Yoshida nutrient solution (Yoshida et al., 1976) in plastic containers (20 plants per line per replication) in a glasshouse situated at the Crop Station, Faculty of Agriculture, Khon Kaen University, Thailand. Plants were grown in Yoshida solution until $21 \mathrm{~d}$ old. For drought treatment, the nutrient solution was replaced by the solution containing polyethylene glycol (PEG6000) to a final concentration of $20 \% \mathrm{w} / \mathrm{v}$. For recovery, the solutions containing PEG were replaced with fresh nutrient solutions. The $\mathrm{pH}$ of nutrient solution was maintained at $5.0-5.5$ during the entire growth period. Controlled plants were grown in Yoshida solution throughout the experiment. The experimental design was completely randomized with 4 replications.

\section{Growth parameters}

On $0,7,14 \mathrm{~d}$ after introduction of PEG solutions and 3 $\mathrm{d}$ after removal of PEG (recovery), shoot and root length of four rice seedlings from each treatment were measured. Shoots and roots were separated and their fresh weights (FW) were directly determined. For dry weight (DW) determination, the shoots and roots were dried at $70^{\circ} \mathrm{C}$ for $48 \mathrm{~h}$.

\section{Leaf water potential (LWP)}

On-site measurement of leaf water potential was performed at midday (12.00-14.00) on young fully expanded leaves using the pressure chamber technique following Turner (1981). The selected leaf was cut to 7-8 $\mathrm{cm}$ in length and immediately placed in the pressure vessel of the Plant Water Status Console (Model 3005, Soil moisture Equipment Corp., USA).

\section{Relative water content ( $R W C$ )}

The remaining portion of the same leaf used for LWP measurement was used for evaluation of RWC and osmotic potential (OP). For RWC, leaves were cut into $2-$ to $3-\mathrm{cm}$ segments before immediately transferred to $1.5 \mathrm{ml}$ preweighed microtubes for measurement of FW. The leaf segments were then transferred to a plastic petri dish $(6 \mathrm{~cm}$ in diameter) containing $10 \mathrm{~mL}$ of deionized water prior to exposure to light from fluorescence tubes for $4 \mathrm{hr}$. Leaf samples were removed from the petri dishes, lightly touched on tissue paper to remove excess water on leaf surface, placed in the same microtubes and reweighed to obtain the turgid weight (TW). Thereafter, the leaf samples were then dried in hot-air oven at $70{ }^{\circ} \mathrm{C}$ for $24 \mathrm{hr}$ or until constant weight was attained to obtain the DW. RWC was determined following the method described by Barrs and Weatherly (1962) with minor modifications, was calculated 
using the equation:

RWC $(\%)=[(F W-D W) /(T W-D W)] \times 100$

where FW is fresh weight, DW is dry weight and TW is turgid weight.

\section{Osmotic potential (OP) and osmotic adjustment $(O A)$}

For the determination of OP, the remaining leaf samples were placed in a zip bagand immersed in liquid nitrogen until they were all frozen. The frozen leaf was used immediately for the determination of OP or stored at -20 ${ }^{\circ} \mathrm{C}$ for later use. The frozen leaf which was left to completely thaw at room temperature was placed in a 1-ml syringe and pressed with the plunger until the sap was expressed through the syringe tip. Ten microliters of the leaf sap was pipetted onto a filter paper disc (5-mm diameter). Then the concentration of solute (osmolality) was measured using a model 5520 osmometer (Wescor Inc., USA) for measurement of osmolality. Leaf osmotic potential was calculated using the van't Hoff equation:

$\mathrm{OP}=-\mathrm{RT} \mathrm{c}$ - where OP represents the osmotic potential $(\mathrm{MPa}), \mathrm{RT}=2.486 \mathrm{~kg} \mathrm{MPa} \mathrm{mol}{ }^{-1}$ at $25^{\circ} \mathrm{C}$, and $\mathrm{c}=$ osmolality of leaf sap $\left(\mathrm{mmol} \mathrm{kg}^{-1} \mathrm{H}_{2} \mathrm{O}\right)$.

The OP at full turgor (osmotic potential at 100\% relative water content, OP100) (Wilson et al., 1979; Flower and Ludlow, 1986; Turner et al., 1986) assuming that apoplastic water content of rice to be $18 \%$ was calculated using the following formula:

OP100 = OP $[(\mathrm{RWC}-18) / 82]-$ where OP is the osmotic potential of the leaf sample and RWC the relative water content of the leaf sample. The OA was calculated from the difference between the OP100 of non-stressed and stressed rice leaves (Flower and Ludlow, 1986):

$\mathrm{OA}=$ non-stressed leaf OP100 - stressed leaf OP100.

\section{Leafproline content}

Proline content was analyzed by the modified procedure of Bates et al. (1973). Approximately $0.1 \mathrm{~g}$ of shoot was homogenized with $5 \mathrm{ml}$ of $3 \%$ aqueous sulfosalicylic acid. Two $\mathrm{ml}$ of extract was reacted with $2 \mathrm{ml}$ of acid ninhydrin and $2 \mathrm{ml}$ of glacial acetic acid and boiled in a water bath at $100{ }^{\circ} \mathrm{C}$ for 1 hour. The reaction was stopped by placing tubes on ice. The solution was extracted with $4 \mathrm{ml}$ of toluene and the absorbance of the toluene fraction was measured at $520 \mathrm{~nm}$. The amount of free proline was evaluated using a standard curve and expressed as $\mu \mathrm{g} \mathrm{g}^{-1}$ tissue fresh weight.

\section{Electrolyte leakage}

Electrolyte leakage was determined as described by Ghoulam et al. (2002) with some modifications. The fully expanded leaf of four plants for each treatment was used. Samples were placed in closed vials containing $10 \mathrm{ml}$ of deionized water for $60 \mathrm{~min}$ at $25^{\circ} \mathrm{C}$. Percent EL of the sample was estimated by measuring the electrical conductivity (EC) of the water after $60 \mathrm{~min}$ (EC60) and after disrupting cell membranes by heating the samples at $100{ }^{\circ} \mathrm{C}$ for $30 \mathrm{~min}$ (ECboil). EL was estimated as follows:

$$
\mathrm{EL}(\%)=(\mathrm{EC} 60 / \mathrm{ECboil}) \times 100 \text {. }
$$

\section{SPAD chlorophyll reading}

Chlorophyll based on SPAD reading was determined on the youngest fully expanded leaf using SPAD-502 chlorophyll meter (Spectrum Technologies Inc., USA) on days 0,7 , and $14 \mathrm{~d}$ after addition of PEG, and $3 \mathrm{~d}$ after recovery.

\section{Statistical analysis}

The data were subjected to analysis of variance using MSTAT-C package (Bricker, 1989). Comparisons of means were made using Duncan's multiple range tests (DMRT) at significant difference $\mathrm{p}<0.05$.

\section{Results}

\section{Analysis of variance}

Analysis of variance showed that differences among lines/cultivar (C), drought treatment (D) and timing (T) were significant for all physiological parameters (Table 1) except for SPAD values which did not show significant between treatment groups. The interactions between lines/cultivar and drought treatment $(\mathrm{C} \times \mathrm{D})$, lines/cultivar and timing $(\mathrm{C} \times \mathrm{T})$ and drought treatment and timing $(\mathrm{D} \times$ T) also significantly affected all physiological parameters except for SPAD values which showed no significant differences in the interaction of $\mathrm{D} \times \mathrm{T}$. Similar trends were observed for interactions of $\mathrm{C} \times \mathrm{D} \times \mathrm{T}$ (Table 1). For OA, the statistical test showed that there were significant differences among lines/cultivar and timing and the interaction between $\mathrm{C} \times \mathrm{T}$ (Table 2). Drought treatment caused significant change in physiological parameters tested in this present study except for SPAD value. The effect of drought was variously depended on genotypes indicating different drought tolerance levels among CSSLs and parents.

Table 1. Mean squares for RWC, LWP, OP, proline, EL, SPAD, FW and DW of 4 rice lines/cultivar treated with drought treatment (20\% PEG) for $0,7,14 \mathrm{~d}$ and $3 \mathrm{~d}$ after recovery

\begin{tabular}{|c|c|c|c|c|c|c|c|c|c|}
\hline $\begin{array}{l}\text { Source of } \\
\text { variation }\end{array}$ & DF & $\begin{array}{c}\text { RWC } \\
(\%)\end{array}$ & $\begin{array}{l}\text { LWP } \\
(\mathrm{MPa})\end{array}$ & $\begin{array}{c}\mathrm{OP} \\
(\mathrm{MPa})\end{array}$ & $\begin{array}{c}\text { Proline } \\
(\mu \mathrm{g} / \mathrm{g} F W)\end{array}$ & $\begin{array}{l}\text { EL } \\
(\%)\end{array}$ & $\begin{array}{l}\text { SPAD } \\
\text { (value) }\end{array}$ & $\begin{array}{c}\text { FW } \\
\text { (g/plant) }\end{array}$ & $\begin{array}{c}\text { DW } \\
\text { (g/plant) }\end{array}$ \\
\hline Lines/cultivar (C) & 3 & $46.67^{*}$ & $0.04154^{*}$ & $0.0419^{*}$ & $706039^{*}$ & $17.864^{*}$ & $38.097^{*}$ & $22.61^{*}$ & $1.5861^{*}$ \\
\hline Treatment (D) & 1 & $3942.72^{*}$ & $3.04428^{*}$ & $11.3169^{*}$ & $6975682^{*}$ & $299.941^{*}$ & $1.734^{\mathrm{ns}}$ & $1092.78^{*}$ & $38.3907^{*}$ \\
\hline Timing (T) & 3 & $1618.94^{*}$ & $0.88898^{*}$ & $4.3370^{*}$ & $4878597^{*}$ & $163.625^{*}$ & $225.465^{*}$ & $265.24^{*}$ & $25.3530^{*}$ \\
\hline $\mathrm{C} \times \mathrm{D}$ & 3 & $57.41^{*}$ & $0.04380^{*}$ & $0.0546^{*}$ & $659729^{*}$ & $26.347^{*}$ & $47.688^{*}$ & $50.37^{*}$ & $0.9968^{*}$ \\
\hline $\mathrm{C} \times \mathrm{T}$ & 9 & $8.83^{*}$ & $0.02504^{*}$ & $0.0170^{*}$ & $438487^{*}$ & $18.746^{*}$ & $5.375^{*}$ & $47.40^{*}$ & $0.1094^{*}$ \\
\hline $\mathrm{D} \times \mathrm{T}$ & 3 & $1154.94^{*}$ & $0.84773^{*}$ & $2.8793^{*}$ & $2157873^{*}$ & $164.084^{*}$ & $1.994^{\mathrm{ns}}$ & $193.39^{*}$ & $8.6460^{*}$ \\
\hline $\mathrm{C} \times \mathrm{D} \times \mathrm{T}$ & 9 & $13.41^{*}$ & $0.02728^{*}$ & $0.0372^{*}$ & $475872^{*}$ & $27.664^{*}$ & $2.028^{\mathrm{ns}}$ & $29.87^{*}$ & $0.2927^{*}$ \\
\hline CV (\%) & & 1.72 & 4.37 & 4.20 & 5.62 & 8.54 & 3.27 & 9.36 & 8.56 \\
\hline
\end{tabular}

ns $=$ Non-significant and ${ }^{*}$ significant at $\mathrm{P} \leq 0.05$, respectively. 
682

Table 2. Mean squares for OA of 4 rice lines/cultivar subjected to

\begin{tabular}{ccc}
\hline Source of variation & DF & OA $(\mathrm{MPa})$ \\
\hline Lines $/$ cultivar $(\mathrm{C})$ & 3 & $0.3330^{*}$ \\
\hline Timing $(\mathrm{T})$ & 2 & $12.7600^{*}$ \\
$\mathrm{C} \times \mathrm{T}$ & 6 & $0.0847^{*}$ \\
$\mathrm{CV}(\%)$ & & 4.20 \\
\hline${ }^{*}$ significant at $\mathrm{P} \leq 0.05$, respectively. & &
\end{tabular}

RWC and LWP were decreasing with increasing period of drought imposed by 20\% PEG supplement in all rice lines/cultivar. At $7 \mathrm{~d}$, the highest decline in RWC was found in the recurrent patent, KDML105 while CSSL1-16, CSSL-18 and DH212 showed no significant changes in RWC compared to those of control groups. At $14 \mathrm{~d}$, RWC was significantly reduced in all rice lines/cultivars, especially in KDML105 and CSSL-18 (decreased 34\% and 31\%, respectively compared to control plants). For CSSL1-16 respectively. During recovery period, all rice lines/cultivar and DH212 were significantly higher than those of CSSL118 and KDML105 (Fig. 1A).

There was no significant difference in LWP of droughtstressed plants at $7 \mathrm{~d}$ whereas a significant difference was observed at $14 \mathrm{~d}$ after drought treatment. Drought severely affected LWP in KDML105 (decreased 83\% compared to control) followed by CSSL1-16, CSSL1-18 and the donor, DH212 (79\%, 75\% and 69\%, respectively). After replacing plants to the normal condition (recovery), it was found that LWP increased in all rice lines/cultivar. DH212 had significantly higher LWP than other lines/cultivars both under stress and recovery conditions (Fig. 1B). drought treatment for 7, 14 and $3 \mathrm{~d}$ after recovery

\section{Changing of water status under drought and recovery} and $\mathrm{DH} 212$, the reductions were $23 \%$ and $27 \%$ were able to increase RWC. However, RWC of CSSL1-16

Osmotic potential $(O P)$ and osmotic adjustment $(O A)$ under drought treatment

For OP, OP of both CSSL1-16 and CSSL1-18 were higher than those of their parental lines at $7 \mathrm{~d}$ of drought stress. At $14 \mathrm{~d}$ of the treatment, the reductions in OPs of CSSL1-16, CSSL1-18, KDML105 and DH212 were 51\%, $53 \%, 54 \%$ and $43 \%$, respectively compared to those of control groups; Fig. 2A). Under drought stress, plants exhibited high OA at 7 d. CSSL1-16 and CSSL1-18 had highest $\mathrm{OA}$ followed by the donor, $\mathrm{DH} 212$ and recurrent parent, KDML105. At $14 \mathrm{~d}$ after the treatment, OA of all lines/cultivar was markedly reduced. CSSL1-16 showed the highest OA in contrast to DH212 which had the lowest. OA of drought-stressed CSSL1-18 and KDML105 were intermediate between CSSL1-16 and DH212. After $3 \mathrm{~d}$ recovery from stress, it was observed that OA still decreased, except for DH212 in which OA increased 2-fold compared to OA of DH212 at $14 \mathrm{~d}$ drought treatment (Fig. 2B).

\section{Proline accumulation, electrolyte leakage and chlorophyll content}

With increasing period of drought treatment, proline accumulation was significantly increased in all lines/cultivar. No significant differences of proline content were observed among stressed plants at $7 \mathrm{~d}$ of the treatment whereas differences in proline content were noted at $14 \mathrm{~d}$. All lines/cultivars showed a significant increase by $76 \%, 47 \%$, $24 \%$ and $52 \%$ from controlled plants on the same day for CSSL1-16, CSSL1-18, KDML105 and DH212, respectively. After $3 \mathrm{~d}$ recovery, proline content in all plants was significantly reduced compared to the plants at $14 \mathrm{~d}$ treatment. The proline content of CSSL1-18 was at the same level as KDML105 but lower than that of CSSL1-16 and DH212 (Fig. 3A).

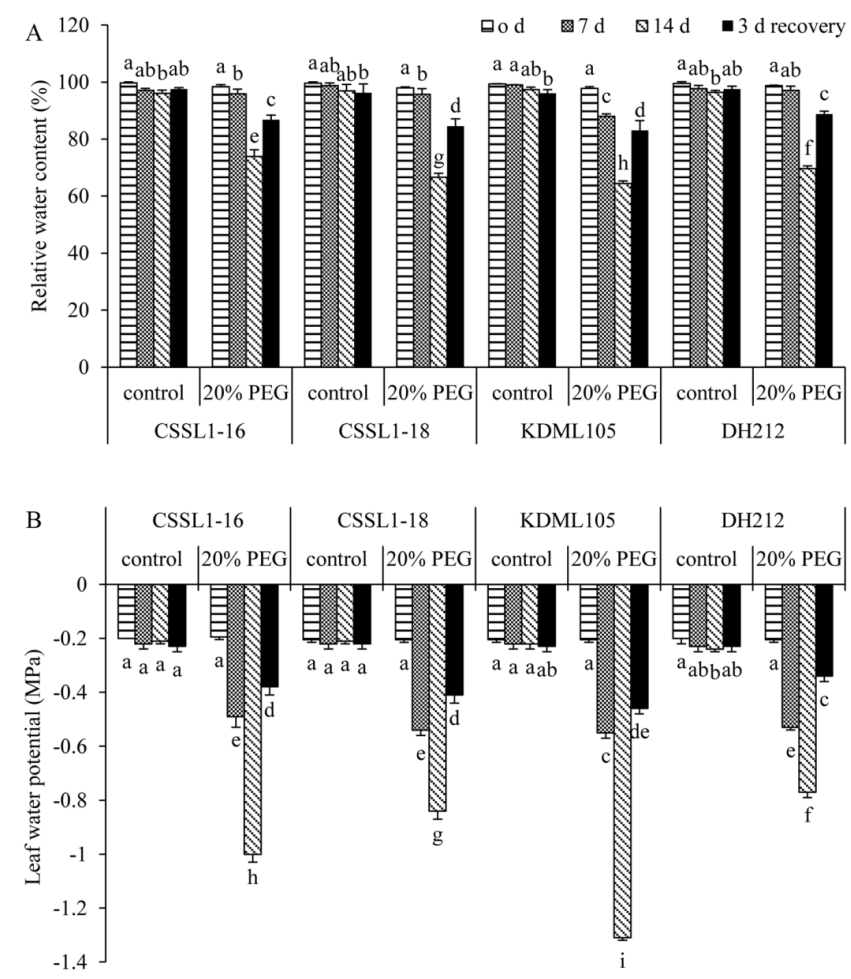

Fig. 1. Relative water content (A) and leaf water potential of rice leaves (B) under control and $20 \%$ PEG-treated for 0, 7, $14 \mathrm{~d}$; and $3 \mathrm{~d}$ after recovery. Values are means of four replications \pm SD 

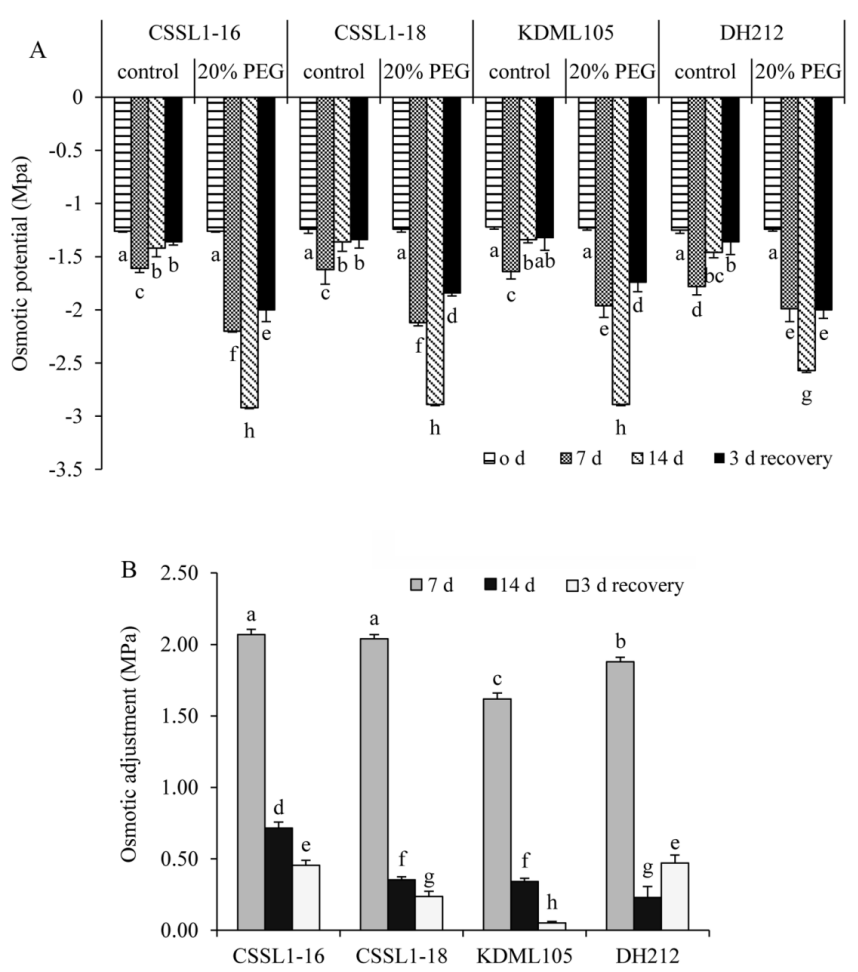

Fig. 2. Osmotic potential of rice leaves under control and 20\% PEG-treated for 0,7, $14 \mathrm{~d}$; and $3 \mathrm{~d}$ after recovery (A), and osmotic adjustment of rice leaves under drought period and $3 \mathrm{~d}$ after recovery (B). Values are means of four replications $\pm \mathrm{SD}$
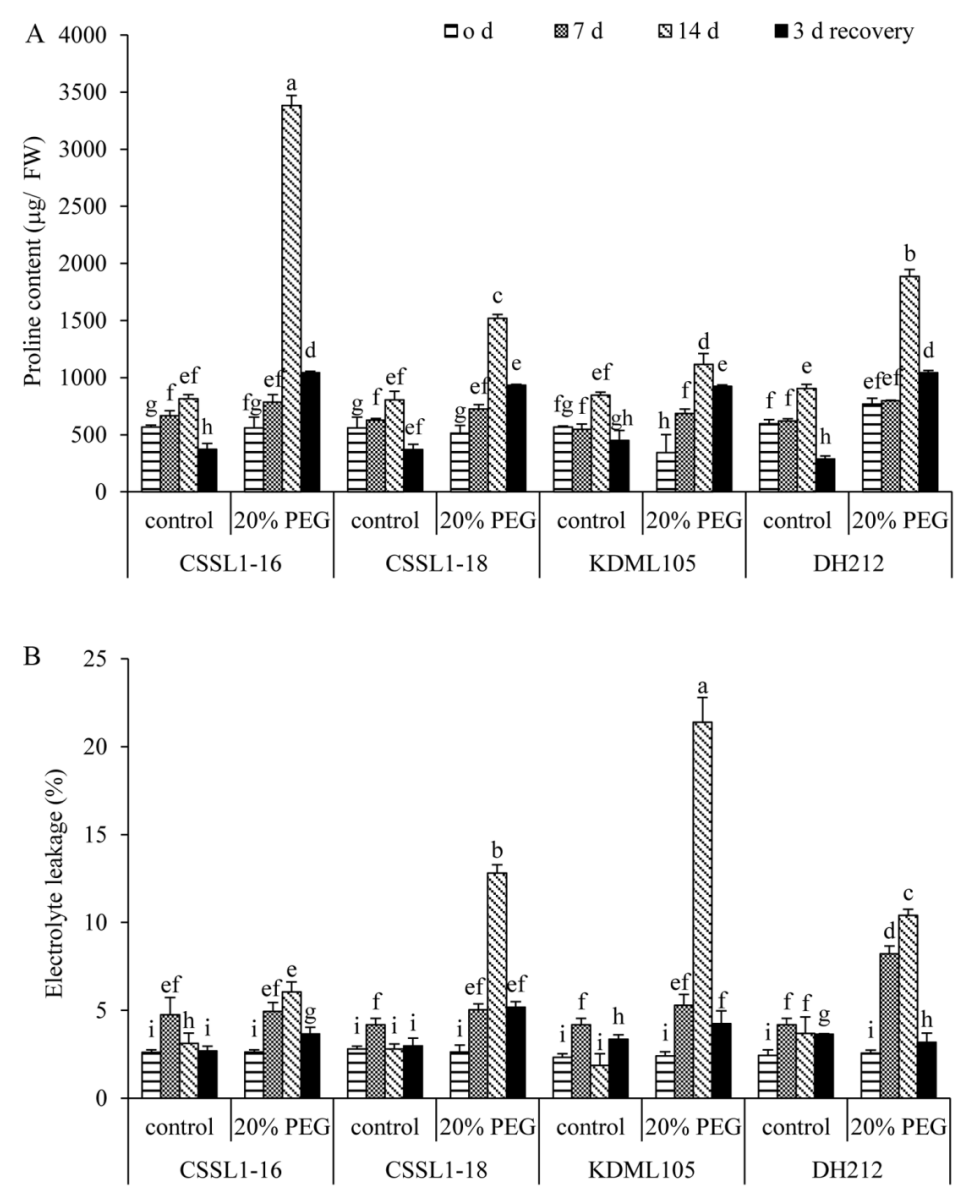

Fig. 3. Proline content (A) and the electrolyte leakage percentage of rice leaves (B) under control and 20\% PEG-treated for 0, 7 , $14 \mathrm{~d}$; and $3 \mathrm{~d}$ after recovery. Values are means of four replications $\pm \mathrm{SD}$ 
684

Similarly to proline, EL increased during the long period of drought treatment. At $7 \mathrm{~d}$ of the treatment, no significant difference was found in EL in all rice lines/cultivar except for the donor, DH212 which showed higher EL than other lines/cultivars. When plants were exposed to drought for 14 $\mathrm{d}$, the most pronounced increase in EL was recorded in KDML105 (increased 91\% compared to controlled plants) followed by CSSL1-18 (78\%), DH212 (65\%) and CSSL116 (48\%), respectively. At recovery period, EL decreased in all rice lines/cultivar in response to growth solution under normal condition. CSSL1-18 and KDML105 had significantly higher EL than CSSL1-16 and DH212 (Fig. $3 \mathrm{~B})$.

For chlorophyll content, it was found that SPAD values increased slightly under drought and recovery periods and SPAD values in all rice lines/cultivars were not significantly different (Fig. 4).

\section{Growth parameters under drought and recovery}

Drought stress resulted in a significant reduction in fresh (Fig. 5A) and dry (Fig. 5B) weights of all rice lines/cultivar. During drought treatment at $7 \mathrm{~d}$ and $14 \mathrm{~d}$, KDML105 showed the highest reduction in fresh weight (decreased $71 \%$ and $81 \%$, respectively compared to those control groups). For other lines (CSSL1-16, CSSL1-18 and DH212), there was approximately $51-56 \%$ reduction in fresh weight at $7 \mathrm{~d}$ after stress treatment. At $14 \mathrm{~d}$ after stress, CSSL1-16 showed the lowest reduction (67\%) followed by CSSL1-18 (75\%) and DH212 (76\%). After $3 \mathrm{~d}$ recovery from stress, fresh weight was significantly increased in all rice lines/cultivar (increased 45\%, 34\%, 52\% and 43\% in CSSL1-16, CSSL1-18, KDML105 and DH212, respectively). However, it was found that CSSL1-16 showed the highest fresh weight followed by CSSL1-18, KDML105 and DH212, respectively.

Similar trend was observed in dry weight (Fig. 5B), a decrease in dry weight has been shown in all rice lines/cultivars. When plants were exposed to drought stress for $7 \mathrm{~d}$, CSSL1-18 showed a slight decrease in dry weight ( $0.4 \%$ compared to controlled plants) while the most pronounced decrease was observed in KDML105 (55\%).
There was $27 \%$ and $31 \%$ reduction in dry weight of CSSL116 and DH212, respectively. At $14 \mathrm{~d}$ drought stress, dry weight of both CSSL1-16 and 18 was reduced (51\% and $54 \%$, respectively compared to those of the control groups) but not as much as those of parental lines (63\% in KDML105 and 60\% in DH212, respectively). Noticeably, only CSSL1-16 was able to recover from drought stress. Dry weight of CSSL1-16 was significantly increased (27\%) compared to those of CSSL1-16 previously supplied with $20 \%$ PEG. In contrast, no significant differences between dry weight of the plants $14 \mathrm{~d}$ of stress and $3 \mathrm{~d}$ of recovery were observed in CSSL1-18, KDML105 and DH212.

\section{Discussion}

It is well-known that drought restricts water supply which results in a reduction of leaf water content and potential (Amini et al., 2014). The water loss can lower leaf water potentials, leading to reduced turgor, stomatal conductance, and photosynthesis, and thus eventually to reduce grain yield (Akbarian et al., 2011; Amini et al., 2014). A significant decline in RWC was clearly observed in KDML105 at $7 \mathrm{~d}$ while in other lines a clear difference between control and treatment groups was found at $14 \mathrm{~d}$ of the treatment. This suggested that drought stress imposed more negative impact on water balance in KDML105 than CSSL1-16, CSSL1-18 and DH212. Screening drought tolerant rice using morpho-physiological traits including RWC, revealed that drought tolerant showed higher RWC than drought sensitive genotypes (Kumar et al., 2014). In addition, in rice, it has been reported that LWP is considered to be an important physiological trait for drought tolerance under water deficit (Jongdee et al., 2002). Similar to RWC, numerous experiments have reported that higher decrease in LWP was observed in drought susceptible genotypes than in the drought tolerant ones in many plant species (Silva et al., 2010; Silvestre et al., 2017). In this present study, LWP in all rice lines/cultivar showed significant decrease between controlled plants and stressed plants at $7 \mathrm{~d}$ of the treatment. However, no consistent

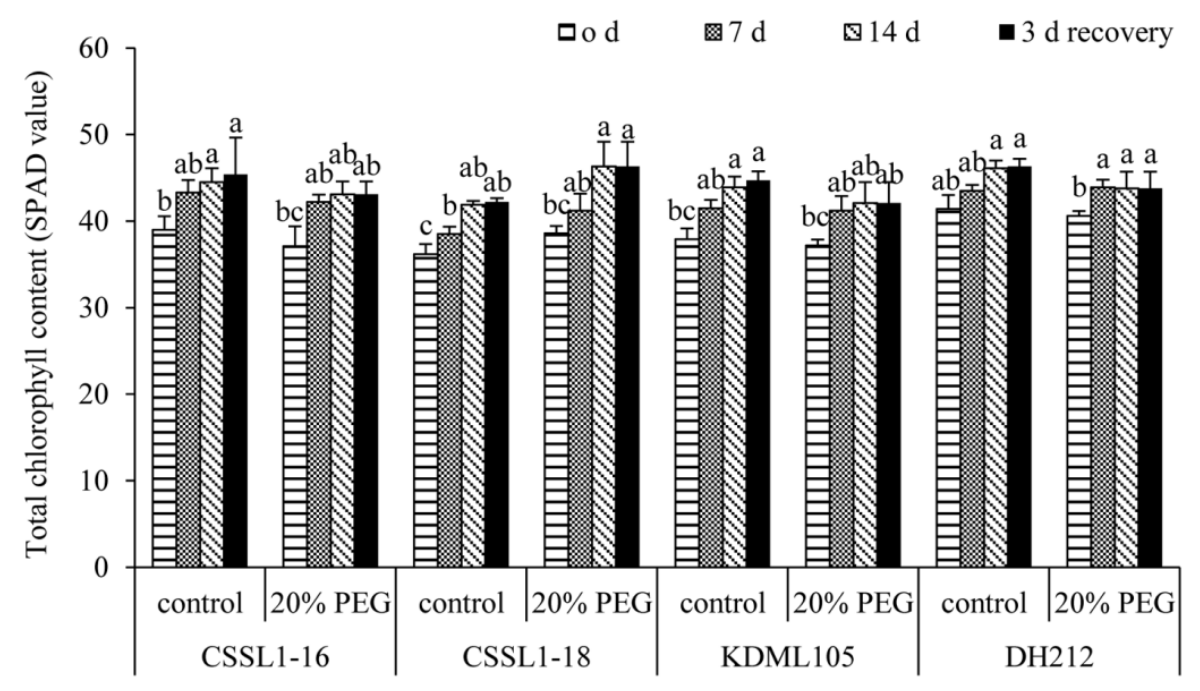

Fig. 4. SPAD chlorophyll meter readings of rice leaves under control and 20\% PEG-treated for 0, 7, $14 \mathrm{~d}$; and $3 \mathrm{~d}$ after recovery. Values are means of four replications \pm SD 

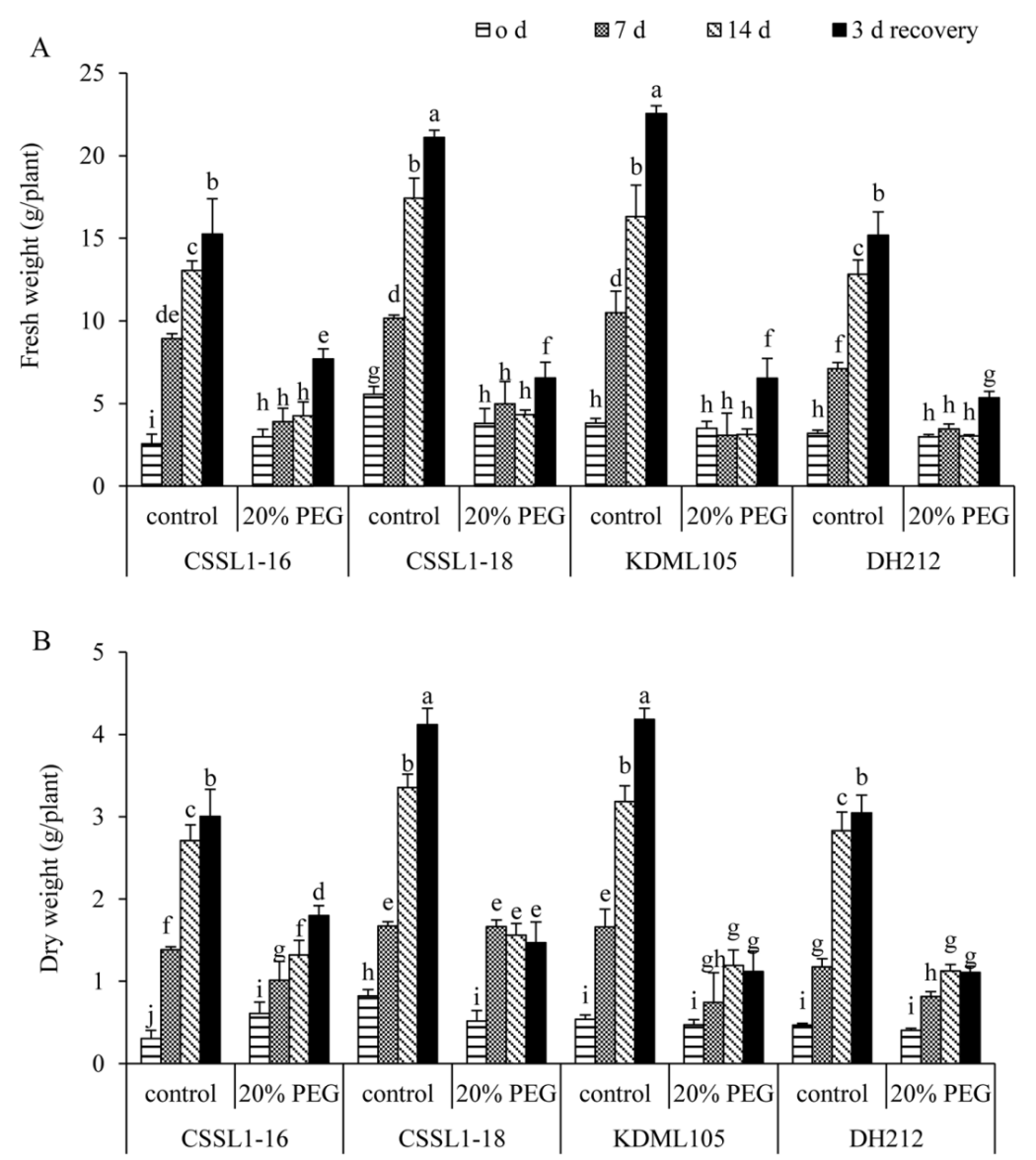

Fig. 5. Fresh weight (A) and dry weight (B) of rice plants under control and 20\% PEG-treated for 0, 7, 14 d; and $3 \mathrm{~d}$ after rewatering (recovery). Values are means of four replications \pm SD

differences in LWP were observed between lines/cultivar. A significant difference among plants was exhibited at long term drought treatment (14 d). The donor, DH212 was recorded the highest value of LWP in contrast to recurrent parent, KDML105 which was noted the lowest. LWP of drought stressed CSSL1-16 and CSSL1-18 was intermediate between their parents. Therefore, determining water status of plants under drought stress condition could be summarized that CSSL1-16 and CSSL1-18 showed their superiority for drought tolerance over KDML105. After removing drought stress, CSSL1-16 still showed better performance to recover from drought stress than KDML105. Based on our data, using RWC might take shorter time for screening drought tolerant rice than using LWP supported by the observation that drought susceptible genotype showed significant reduction in RWC at earlier period of drought applied.

Maintaining OP at low level in plant cells caused by accumulation of osmolytes is a part of osmoregulation which is necessary for plant adaptation to drought stress (Zivcak et al., 2016). Under prolonged drought treatment, only drought tolerant donor, $\mathrm{DH} 212$ showed significantly lower OP than those of other lines/cultivar. Similar finding was observed in two contrasting rice cultivars under drought in which the authors reported that drought tolerant cultivar showed less reduction in OP than drought sensitive ones (Khan et al., 2017). OA is considered to be an important mechanism for drought tolerance ( $\mathrm{Xu}$ et al.,
2010). Accumulation of compatible solutes such as proline is believed to enhance $\mathrm{OA}$ and decrease $\mathrm{OP}$ and promote water uptake into plant cells under water deficit (Zivcak et al., 2016; Silvestre et al., 2017). At the maximum period of drought stress, CSSL1-16 accumulated highest proline which was associated with highest $\mathrm{OA}$ in contrast to KDML105. The large amount of proline level in CSSL1-16 might be involved in enhancing OA which could enhance drought tolerant ability as compared to its recurrent parent, KDML105. For CSSL1-18 and DH212, the association between OA and proline accumulation remained elusive. However, occurrence of OA under stress condition not only depends on proline but also depends on other organic osmolytes such as sugar and inorganic ions such as potassium ions (Silva et al., 2010; Zivcak et al., 2016). After plants recovered from stress, only DH212 could increase OA. However, OA of CSSL1-16 was significantly higher than KDML105 suggesting that CSSL1-16 had higher ability to recover from stress than KDML105.

Membrane damage caused by ROS generally occurs when plants are exposed to abiotic stress including drought. During stress period, CSSL1-16 and DH212 showed less increase in EL (an indicator for membrane damage) than CSSL1-18 and KDML105 which showed highest EL. These results indicated that the effect of drought stress was more pronounced in KDML105 which is drought sensitive cultivar in contrast to CSSL1-16. Furthermore, it has been reported that proline could act as ROS scavenging 
686

molecules (Liang et al., 2013). The lowest and highest EL detected in CSSL1-16 and KDML105 respectively might be associated with proline accumulated in those plants under drought. SPAD index was used in this study to determine chlorophyll content. It is obvious that chlorophyll content tended to be similar in all rice lines/cultivar. Therefore, chlorophyll content via SPAD index could not be an appropriate indicator to evaluate drought tolerant rice in this study.

Exposure of 20\% PEG resulted in reduction in fresh and dry weights of all rice lines/cultivar. However, fresh weight under stress period in all plants did not show any significant differences. During recovery, it was found that all plants were able to recover from drought stress based on fresh weight. For dry weight at 7 and 14 d drought stress, CSSL118 had highest dry weight than other rice lines/cultivar. At $14 \mathrm{~d}$, both CSSL1-16 and CSSL1-18 had significantly higher dry weight than their parental lines. It can be concluded that CSSL1-16 and CSSL1-18 had better growth under drought than KDML105. These results are consistent with many reports which presented that drought tolerant genotypes could maintain higher growth than sensitive ones (Kumar et al., 2014; Mejri et al., 2016). Notably, only CSSL1-16 was able to recover from drought stress as indicated by a significant increase dry weight. This finding suggests that CSSL1-16 had better adaptive mechanisms for alleviating negative effects from drought stress than other rice lines/cultivars to resume better growth than other rice lines/cultivar.

\section{Conclusions}

Based on comparative physiological responses of 2 CSSLs and their parental lines under drought stress, it was found that introgression of DT-QTL segments from the donor; DH212 could enhance drought tolerance in the resultant lines compared to the recurrent parent, KDML105. Drought tolerance mechanisms of CSSL lines demonstrated in this study included osmotic adjustment and the ability to maintain water status under drought stress.

\section{Acknowledgements}

This work was financially supported by the National Center for Genetic Engineering and Biotechnology (BIOTEC), National Science and Technology Development Agency (NSTDA). A scholarship from the Thailand Graduate Institute of Science and Technology (TGIST) awarded to PL is gratefully acknowledged. NN has received scholarship under the Post-Doctoral Training Program from Research Affairs and Graduate School, Khon Kaen University, Thailand (Grant no. 58331).

\section{References}

Amini H, Arzani A, Karami M (2014). Effect of water deficiency on seed quality and physiological traits of different safflower genotypes. Turkish Journal of Biology 38:271-282.

Akbarian A, Arzani A, Salehi M, Salehi M (2011). Evaluation of triticale genotypes for terminal drought tolerance using physiological traits. Indian Journal of Agricultural Sciences 81:1110-1115.
Barrs HD, Weatherly PE (1962). A re-examination of the relative turgidity technique for estimating water deficits in leaves. Australian Journal of Biological Sciences 15:413-428.

Bates LS, Waldren RP, Teare ID (1973). Rapid determination of free proline for water stress studies. Plant and Soil 39:205-207.

Bouman BAM, Peng S, Castaòeda AR, Visperas RM (2005). Yield and water use of irrigated tropical aerobic rice systems. Agricultural Water Management 74:87-105.

Bricker AA (1989). MSTAT-C user's guide. Michigan State University, Michigan.

Farooq M, Bramley H, Palta JA, Siddique KHM (2011). Heat stress in wheat during reproductive and grain filling phases. Critical Reviews in Plant Sciences 30:491-507.

Farooq M, Hussain M, Wahid A, Siddique KHM (2012). Chapter 1 Drought Stress in Plants: An Overview. In: Aroca R (Ed). Plant Responses to Drought Stress. Springer-Verlag, Heidelbergpp 1-33.

Flower DJ, Ludlow MM (1986). Contribution of osmotic adjustment to the dehydration tolerance of water-stressed pigeon pea (Cajanus cajan (L. millsp.)) leaves. Plant, Cell \& Environment 9:33-40.

Ghoulam C, Foursy A, Fares K (2002). Effects of salt stress on growth, inorganic ions and proline accumulation in relation to osmotic adjustment in five sugarbeet cultivars. Environmental and Experimental Botany 47:39-50.

Jongdee B, Fukai S, Cooper M (2002). Leaf water potential and osmotic adjustment as physiological traits to improve drought tolerance in rice. Field Crops Research 76:153-163.

Kanjoo V, Punyawaew K, Siangliw JL, Jearakongman S, Vanavichit A, Toojinda T (2012). Evaluation of agronomic traits in chromosome segment substitution lines of KDML105 containing drought tolerance QTL under drought stress. RiceScience 19:117-124.

Khan F, Upreti P, Singh R, Shukla PK, Shirke PA (2017). Physiological performance of two contrasting rice varieties under water stress. Physiology and Molecular Biology of Plants 23:85-97.

Kumar S, Dwivedi SK, Singh SS, Jha SK, Lekshmy S, Elanchezhian R, ... Bhatt BP (2014). Identification of drought tolerant rice genotype by analysing drought tolerance indices and morpho-physiological traits. SABRAOJournal of Breeding and Genetics 46:217-230.

Lonbani M, Arzani A (2011). Morpho-physiological traits associated with terminal drought stress tolerance in triticale and wheat. Agronomy Research 9:315-329.

LiangX,Zhang L, Natarajan SK, Becker DF (2013). Proline mechanisms of stress survival. Antioxidants \& Redox Signaling 19:998-1011.

Mejri M, Siddique KHM, Saif T, Abdelly C, Hessini K (2016). Comparative effect of drought duration on growth, photosynthesis, water relations, and solute accumulation in wild and cultivated barley species. Journal of Plant Nutrition and Soil Science 179:327-335.

Saha P, Sade N, Arzani A, Rubio Wilhelmi MDM, Coe KM, Li B, Blumwald E (2016). Effects of abiotic stress on physiological plasticity and water use of Setaria viridis(L.). PlantScience 251:128-138.

Silva EN, Ferreira-Silva SL, Viégas RA, Silveira JAG (2010). The role of organic and inorganic solutes in the osmotic adjustment of droughtstressed Jatropha curcas plants. Environmental and Experimental Botany 69:279-285.

Silvestre WVD, Silva PA, Palheta LF, Neto CFO, Souza RORM, Festucci- 
Buselli RA, Pinheiro HA (2017). Differential tolerance to water deficit in two açaí (Euterpe oleracea Mart.) plant materials. Acta Physiologiae Plantarum 39:4.

Turner NC (1981). Techniques and experimental approaches for the measurement of plant water status. Plant and Soil 58:339-366.

Turner NC, O’Toole JC, Cruz RT, Yambao EB, Ahmad S, Namuco OS, Dingkuhn M (1986). Response of seven diverse rice cultivars to water deficit. II. Osmotic adjustment, leaf elasticity, leaf extension, leaf death, stomata conductance and photosynthesis. Field Crops Research 13:273286.

Wilson JR, Fischer MJ, Schulze ED, Dolby GR, Ludlow MM (1979). Comparison between pressure-volume and dew point hygrometry techniques for determining the water relations characteristics of grass and legumeleaves. Oecologia41:77-88.
Xu Z, Zhou G, Shimizu H (2010). Plant responses to drought and rewatering. Plant Signaling \& Behavior 5:649-654.

YoshidaS, Forno DA, CockJH, Gomez KA (1976). Laboratory manual for physiological studies of rice. International Rice Research Institute, Los Banos.

Zivcak M, Brestic M, SYTAR O (2016). Osmotic adjustment and plant adaptation to drought stress. In: Hossainh MA, Wani SH, Bhattacharjee S, Burritt DJ, Tran LSP (Eds). Drought Stress Tolerance in Plants Vol 1. Springer International Publishing, Switzerland pp 105143.

Zu X, Lu Y, Wang Q, Chu P, Miao W, Wang H, La H (2017). A new method for evaluating the drought tolerance of upland rice cultivars. The CropJournal 5:488-498. 